\title{
Holding a MIRror up to the robustness of the prostate cancer urinary transcriptome
}

\author{
Adele E. Connor ${ }^{1,2 \#}$, Asia C. Jordan ${ }^{1,2 \#}$, Antoinette S. Perry ${ }^{1,2}$ \\ ${ }^{1}$ School of Biology and Environmental Science, University College Dublin, Dublin, Ireland; ${ }^{2}$ Cancer Biology and Therapeutics Laboratory, Conway \\ Institute of Biomedical and Biomolecular Research, University College Dublin, Dublin, Ireland \\ \#These authors contributed equally to this work. \\ Correspondence to: Dr. Antoinette Perry. Room 2.02 Science West, O’Brien Science Centre, UCD, Dublin 4, Ireland. Email: Antoinette.perry@ucd.ie. \\ Provenance: This is an invited article commissioned by the Section Editor Dr. Xiao Li, MD (Department of Urology, Jiangsu Cancer Hospital \& \\ Jiangsu Institute of Cancer Research \& Nanjing Medical University Affiliated Cancer Hospital, Nanjing, China). \\ Comment on: Jeon J, Olkhov-Mitsel E, Xie H, et al. Temporal stability and prognostic biomarker potential of the prostate cancer urine transcriptome. \\ J Natl Cancer Inst 2019. [Epub ahead of print].
}

Submitted Sep 06, 2019. Accepted for publication Sep 18, 2019.

doi: $10.21037 /$ tau. 2019.09 .25

View this article at: http://dx.doi.org/10.21037/tau.2019.09.25

There is great interest in the field of liquid biopsies across the field of oncology. They hold exciting promise as a tool to study circulating tumour cells, nucleic acids and extracellular vesicles, which may aid cancer screening/early detection, monitoring response to therapies and detecting minimal residual disease. Urine is a particularly attractive non-invasive liquid biopsy for urological malignancies, due to its ease of (serial) collection and direct proximity to primary tumour cells of the prostate, bladder and kidney.

In prostate cancer, the field of urine biomarkers is vibrantly active. Several urinary tests are already available for patients and doctors to aid risk-stratification and guide decision to biopsy (1-3). Notably, these all operate by measuring the expression of a small number of protein coding genes. The advent of Movember's Global Action Plan on urine biomarkers (GAP1) led to the international co-ordinated development of several other urine biomarker panels for high-grade prostate cancer that were recently described, namely epiCaPture, PUR and Procure (4-6).

One of the most attractive features of liquid biopsies, such as urine, is the "holistic" nature of the analyte. Prostate cancer boasts extreme molecular heterogeneity, with frequencies of individual gene point mutations typically $<13 \%$ (7). Structural rearrangements such as the TMRPSS2-ERG gene fusion are more common, found in $\sim 50 \%$ cases (8), whilst certain epigenetic alterations (such as hypermethylation of GSTP1) are more prevalent again, detected in $>80 \%$ of tumours (9). This heterogeneity, together with the multi-focal nature of prostate cancer and the sampling bias of needle biopsies, pose a significant hurdle for tissue-based prognostic assays. Could liquidbased biomarkers provide the solution? Perhaps. However, we require more certainty around the reproducibility and intra-individual robustness of liquid biopsy (urine) biomarkers.

This study by Jeon and colleagues goes some way to tackling this very complex issue by evaluating the temporal stability of urine biomarkers. Focusing on microRNAs (miRs), they longitudinally profiled the expression of 673 miRs (at two time points several months apart) in a small cohort of men with low-risk prostate cancer to answer the questions of i) stability within individuals (intra-individual) and ii) variability between individuals (inter-individual), over time. Unsurprisingly, the authors found that urinary miR expression profiles were more similar within individuals than between individuals. The main difference between different urine samples collected from the same man was the absolute number of miRs counted, rather than subtle expression changes over time. The authors next considered the relationship of genomic location on the spatial variability of miR expression. This is relevant because there are multiple examples of $\mathrm{miR}$ gene clusters, which encode miRs that co-ordinately regulate cellular processes, such as miR-15a-16-1 (10). Notably, it was observed that the most intrastable miRs were localised to copy number variation (CNV) neutral loci, such as regions of chromosomes 
6 and 17. Intrastable miRs were also predicted to have more mRNA targets than intravariable miRs, although the significance of this remains unknown; the biological functions of predicted target genes were mostly the same between intrastable and -variable miRs.

The authors used this knowledge of the most stable and robustly detectable miRs in the urine of men with lowrisk prostate cancer to inform the design of a urine miR biomarker panel for aggressive disease. They hypothesised that the most intrastable miRs could serve as potential prognostic biomarkers to risk-stratify patients into low- and high-risk disease. In a second cohort of 99 men, with lowrisk, Gleason 6 disease $(n=49)$ and high-risk Gleason $>7$ $(\mathrm{n}=50)$, they identified 6 miRNA, whose urinary expression could differentiate between high-risk and low-risk disease, and which largely consisted of intrastable miRs. Using cross validation and feature selection, they built prognostic models of aggressive disease, which they validated in a small third cohort. The model consisting of 7 intrastable miRs (miR-3195, let-7b-5p, miR-144-3p, miR-451a, miR-148a$3 \mathrm{p}, \mathrm{miR}-512-5 \mathrm{p}$, and miR-431-5p) was found to distinguish between low- and high-risk disease at an AUC of 0.74, which outperformed all other models that consisted of either intervariable or randomly generated $7 \mathrm{miR}$ panels. Finally, it was shown that urinary detection of the $7 \mathrm{miR}$ panel was highly correlated with presence in matched primary tumour in nine men.

In conclusion, Jeon et al. employed urinary miR transcriptomics to show that microRNAs could serve as a liquid biopsy tumour surrogate to non-invasively distinguish between aggressive and non-aggressive prostate cancer. Utilizing three different cohorts of patients, in addition to analysis with TCGA data, the study produced a $7 \mathrm{miR}$ panel prognostic for high-risk prostate cancer. However, their validation cohort consisted of only 11 high-grade patients and thus caution should be taken not to over-interpret the potential prognostic utility of these findings. Other prognostic miR panels have previously been reported in liquid biopsies (11-15). What distinguishes this study is the experimental design; most other studies inform their selection of a handful of candidate prognostic miRs purely on tumour tissue profiling. This study includes two important advantages. Firstly, the urinary transcriptome was profiled, albeit in a small group of clinically homogenous men. This omic style approach showed that broadly speaking, urinary miR transcriptomes of men with low-risk prostate cancer are representative of those miR expression patterns observed in low-risk tumours $(80 \%$ of miRs detected in at least one urine sample were observed in lowrisk tumours from TCGA). Further work in a much larger and (physiologically, pathologically, ethnically) diverse cohort is needed to fully landscape the urinary miRNA transcriptome and the relative contributions from different anatomical sources. Secondly, and unique to this study design, was the inclusion of serial sampling of urines to allow measurement of temporal stability of miR abundance in individuals over time. Although only two time points were available for all but one man, this study shows the value of this knowledge in the selection of high-performing prognostic biomarkers. Indeed the findings suggest that such a consideration should be factored into the design of all liquid biopsy biomarkers.

The report also raises some interesting questions. It is quite intriguing how miRNAs robustly detected in the urine of men with low-risk prostate cancer and on Active Surveillance are highly discriminatory between low- and high-risk disease. It is unclear whether the $7 \mathrm{miR}$ panel is a signature of low-risk disease. It would be interesting to take the alternative approach and to measure the temporal urinary stability and reproducibility of bonefide microRNAs of aggressive prostate cancer. A further question regarding reproducibility of urine biomarker data surrounds the need for a digital rectal examination (DRE). The prostate DRE is renowned for inter-subject variability in terms of performance and interpretation. This study, as with most other urinary prostate cancer biomarkers, utilised post-DRE samples, because it is a valuable means to increase the presence of prostate cells in urine. It is unclear if all subjects in this study were subject to DRE performed by one individual and what contribution, if any, this made to the robust longitudinal detection of miRs within individuals. It is difficult to imagine how interoperable DRE can be standardised and yet it is an almost obligatory feature of most prostate cancer urine biomarkers.

This work by Jeon et al. nicely demonstrates that liquid biopsy investigators all need to consider the reproducibility of their potential biomarkers. The prognostic value of the specific $7 \mathrm{miR}$ panel for risk stratification of early stage prostate cancer will need to be proven in much larger independent studies.

\section{Acknowledgments}

Funding: The authors would like to acknowledge grant funding from Enterprise Ireland (CF-2018-0894-P), which is supporting their work in this field. 


\section{Footnote}

Conflicts of Interest: The authors have no conflicts of interest to declare.

Ethical Statement: The authors are accountable for all aspects of the work in ensuring that questions related to the accuracy or integrity of any part of the work are appropriately investigated and resolved.

\section{References}

1. Van Neste L, Hendriks RJ, Dijkstra S, et al. Detection of High-grade Prostate Cancer Using a Urinary Molecular Biomarker-Based Risk Score. Eur Urol 2016;70:740-8.

2. Hessels D, Klein Gunnewiek JM, van Oort I, et al. DD3(PCA3)-based molecular urine analysis for the diagnosis of prostate cancer. Eur Urol 2003;44:8-15; discussion 15-6.

3. McKiernan J, Donovan MJ, O'Neill V, et al. A Novel Urine Exosome Gene Expression Assay to Predict Highgrade Prostate Cancer at Initial Biopsy. JAMA Oncol 2016;2:882-9.

4. O'Reilly E, Tuzova AV, Walsh AL, et al. epiCaPture: A Urine DNA Methylation Test for Early Detection of Aggressive Prostate Cancer. JCO Precis Oncol. 2019;2019. doi: 10.1200/PO.18.00134.

5. Connell SP, Hanna M, McCarthy F, et al. A Four-Group Urine Risk Classifier for Predicting Outcome in Prostate Cancer Patients. BJU Int 2019. [Epub ahead of print].

6. Zhao F, Olkhov-Mitsel E, Kamdar S, et al. A urine-based DNA methylation assay, ProCUrE, to identify clinically

Cite this article as: Connor AE, Jordan AC, Perry AS. Holding a MIRror up to the robustness of the prostate cancer urinary transcriptome. Transl Androl Urol 2019;8(Suppl 5):S488-S490. doi: 10.21037/tau.2019.09.25 significant prostate cancer. Clin Epigenetics 2018;10:147.

7. Barbieri CE, Baca SC, Lawrence MS, et al. Exome sequencing identifies recurrent SPOP, FOXA1 and MED12 mutations in prostate cancer. Nat Genet 2012;44:685-9.

8. Tomlins SA, Bjartell A, Chinnaiyan AM, et al. ETS gene fusions in prostate cancer: from discovery to daily clinical practice. Eur Urol 2009;56:275-86.

9. Yegnasubramanian S KJ, Gonzalgo ML, Zahurak M, Piantadosi S, Walsh PC, Bova GS, De Marzo AM, Isaacs WB, Nelson WG. Hypermethylation of CpG islands in primary and metastatic human prostate cancer. Cancer Res 2004;64:1975-86.

10. Bonci D, Coppola V, Musumeci M, et al. The miR-15amiR-16-1 cluster controls prostate cancer by targeting multiple oncogenic activities. Nat Med 2008;14:1271-7.

11. Fendler A, Stephan C, Yousef GM, et al. The translational potential of microRNAs as biofluid markers of urological tumours. Nat Rev Urol 2016;13:734-52.

12. Weber JA, Baxter DH, Zhang S, et al. The microRNA spectrum in 12 body fluids. Clin Chem 2010;56:1733-41.

13. Fredsøe J, Rasmussen AKI, Thomsen AR, et al. Diagnostic and Prognostic MicroRNA Biomarkers for Prostate Cancer in Cell-free Urine. Eur Urol Focus 2018;4:825-33.

14. Rodríguez M, Bajo-Santos C, Hessvik NP, et al. Identification of non-invasive miRNAs biomarkers for prostate cancer by deep sequencing analysis of urinary exosomes. Mol Cancer 2017;16:156.

15. Bryant RJ, Pawlowski T, Catto JW, et al. Changes in circulating microRNA levels associated with prostate cancer. Br J Cancer 2012;106:768-74. 